\title{
State of the art of actuator and damper for micro- processor controlled prosthetic knee
}

\begin{abstract}
Prosthetic knee is the most important component of lower limb prosthesis. Microprocessor-controlled prosthetic knee enables amputee to walk with different velocities without the need to change their gait pattern. This article summarizes and classifies the actuator and damper for micro-processor controlled prosthetic knee. By reviewing the literature in the field and by identifying different scientific research lines that have brought out through the years. To provide the information about possible outcomes in the near future. Summarized data can be used as a reference to develop new prototypes. Further, the overview of state-of-the-art can provide possible solutions to improve the imitation of nature of intelligent prosthetic knee.
\end{abstract}

Keywords: microprocessor-controlled prosthetic knee, amputee, actuator, damper, state-of-the-art
Volume 5 Issue 2 - 2019

\author{
Wujing Cao, ${ }^{1,2}$ Hongliu Yu, ${ }^{1,2}$ Yongbing Lv, ${ }^{3}$ \\ Weiliang Zhao, ${ }^{1,2}$ Qiaoling Meng ${ }^{1,2}$ \\ 'Rehabilitation Engineering and Technology Institute, University \\ of Shanghai for Science and Technology, China \\ ${ }^{2}$ Shanghai Engineering Research Center of Assistive Devices, \\ China \\ ${ }^{3}$ Shanghai Artificial Limb Factory, China
}

Correspondence: Wujing Cao, University of Shanghai for Science and Technology, No.516, Jungong Road, Yangpu District,

Shanghai, China, Email caowujing414@।26.com

Received: November 08, 2017 | Published: April 0I, 2019

\section{Introduction}

According to the data calculation of the second national handicapped person sampling survey, the sum of disabled people in China is 82.96 million, including 24.12 million physical disabilities and 2.26 million amputees. ${ }^{1}$ The number of lower limb amputee is about 1.58 million. ${ }^{2}$ To cope with daily life activities, lower limb amputees require to install lower limb prosthesis. Lower limb prosthesis for a person with transfemoral (above-knee) limb loss includes, at a minimum, a socket, knee, foot, and the necessary pylons or adapters to connect these components. ${ }^{3}$ The part that has taken the biggest efforts in its development is the knee prosthesis. The knee performs a key role during the whole gait cycle, and this is basically due to the fact that the knee joint sets a leg made of two bodies, articulated between each other through it. This is the case in human body between thigh and shank, being this leg configuration from which it is understood a natural gait. Moreover, this last feature, leaving aside damping and impulse functions of feet, is what makes human gait the most efficient mode of moving human body from one place to another. ${ }^{4}$ The prosthetic knee is separated by the complexity of their control: mechanically passive, microprocessor controlled passive, and microprocessor controlled active. ${ }^{5}$ Damping of mechanically passive control knee cannot be changed with different speeds and often characterized by abnormal gait, increasing energy expenditure of amputees to walk. Microprocessor-controlled prosthetic knee are the research focus. The classification of microprocessor-controlled prosthetic knee is shown in Figure 1.

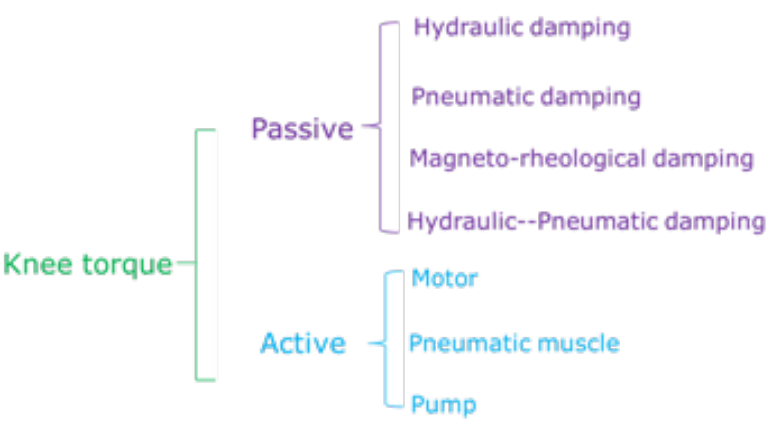

Figure I Classification of microprocessor-controlled prosthetic knee.

\section{Discussion}

Intelligent prosthesis is featured by having an on-board microprocessor which controls the actuator response. The actuator, in the passive ones may be a hydraulic or pneumatic cylinder controlled by opening or closing its valves through servomotors. Yu et al. ${ }^{6}$ designed a double piston hydraulic cylinder for prosthesis. ${ }^{6} \mathrm{Li}$ Wei et.al designed a new hydraulic damping device which the damping was adjusted by rotating the valve stem. But the adjustment of the flexion and extension damping was not independent. ${ }^{7}$ Seid designed a passive controller for hydraulic damper for swing phase of single axis knee; however, the controller resulted in a very large deviation of knee flexion angle from the normal one and hence the designed controller was reported to perform poorly in terms of ground clearance. ${ }^{8}$ Orhanli studied the finite state machine to control intelligent pneumatic knee in different phases through the comparison of physiological gait and prosthetic gait to evaluate the performance of the prosthesis. ${ }^{9}$ For the active ones, flexion and extension of knee are driven by an external force such as the motor, micro pump. ${ }^{10}$ Zhang Fan et al. put forward a kind of powered prosthesis using ball screw. ${ }^{11}$ Frank Sup et.al designed a knee and ankle joint prosthesis, knee and ankle were driven by two motors through ball screw. ${ }^{12}$ Budaker et al. ${ }^{13}$ studied a powered knee joint by gear transmission. ${ }^{13}$ Garrett et.al presented the investigation results of applying the pneumatic artificial muscle actuation to aboveknee prostheses..$^{14}$ Martin et al. ${ }^{15}$ researched the application of hybrid zero dynamics control to powered lower-limb prostheses. ${ }^{15}$

Micro-processor controlled prosthetic knee is a kind of typical human-machine integration robot. Current research on microprocessor controlled prosthetic knee had mostly concerned with passive- or active-only knee prosthesis. These prostheses cannot reproduce the mixing mode (known as hybrid control) of an actual human knee joint. BGA Lambrecht presented a semi-active prosthetic knee. Operation modes includes an active mode driven by a pump, and a passive mode controlled by a variable position valve. ${ }^{16}$ It can mimic the working mechanism of knee joint more naturally. The hybrid control knee prosthesis may be a significant step forward with respect to design the prosthetic knee.

\section{Conclusion}

Microprocessor-controlled passive prosthetic knee are capable of 
altering their behavior instantaneously by means of microprocessor technology. However, they remain limited to generate resisting forces. The active force is still provided by residual limb. Microprocessorcontrolled active prosthetic knee provide external power through motors, pump or other power sources. While they over greater performance and greater functionality, they represent the system with the highest complexity. The size is often very large and the weight is not suitable to wear. The hybrid control prosthetic knee simulating normal knee joint including motor-damper or micro pump-damper is the important research direction.

\section{Acknowledgments}

The work reported in this paper is supported by National Natural Science Foundation of China, number: 61473193 and Shanghai Engineering Research Center of Assistive Devices, number: 15DZ2251700.

\section{Conflicts of interest}

Author declares that no conflict of interest exists.

\section{References}

1. Zhao Yanchao. China disabled persons' federation release the latest disabled population data. Disability in China. 2012;4:3-5.

2. Yang Peng, Liu Zuojun, Geng Yanli. Research advance on key technology of intelligent lower limb prosthesis. Journal of Hebei University of Technology. 2013;1(1):76-80.

3. Le VT, Ohnishi K, Otsuka H, et al. A Method to Analyze Dynamics Properties of Transfemoral Prosthesis. 2016;40:02026.

4. Khan SS, Khan SJ, Usman J. Effects of toe-out and toe-in gait with varying walking speeds on knee joint mechanics and lower limb energetic. Gait \& Posture. 2017;53:185-192.

5. Rosa MD. Controller design and implementation for a powered prosthetic knee. Dissertations \& Theses -Gradworks. 2012.
6. Yu HL, Ling S, Hu JH. Design of electronically controlled hydraulic damper for prosthetic knee. Journal of Clinical Rehabilitative Tissue Engineering Research. 2009;13(39):7635-7638.

7. Li Wei. Working mechanism research and prototype design of intelligent prosthetic knee joint. Southeast University. 2012.

8. Seid S, Sujatha S, Chandramohan S. Design of controller for single axis knee using hydraulic damper. IEEE. 2015. p. 1-5.

9. Orhanli T, Yilmaz A. Application and comparison of finite state control of above-knee prosthesis with pneumatic cylinder at different phases. Signal Processing and Communications Applications Conference. 2015. p. 2234-2237.

10. Gu Hong, Li Weida, Li Juan. The present situation and development trend of the artificial knee prosthesis [J]. Chinese Journal of Rehabilitation Theory and Practice. 2016;22(9):1080-1085.

11. Zhang F, Liu M, Huang H. Preliminary study of the effect of user intent recognition errors on volitional control of powered lower limb prostheses. Conf Proc IEEE Eng Med Biol Soc. 2012;2768-2771.

12. Sup F, Varol HA, Goldfarb M. Upslope walking with a powered knee and ankle prosthesis: initial results with an amputee subject. IEEE Transactions on Neural Systems \& Rehabilitation Engineering. 2011;19(1):71.

13. Budaker B. Active driven prosthesis using a bevel helical gearbox in combination with a brushless dc-motor. Biomedical Engineering. 2012;57(Suppl 1):1096-1096.

14. Waycaster G, Wu SK, Shen X. Design and control of a pneumatic artificial muscle actuated above-knee prosthesis. Journal of Medical Devices. 2011;5(3):031003.

15. Martin AE, Gregg RD. Hybrid Invariance and Stability of a Feedback Linearizing Controller for Powered Prostheses. Proc Am Control Conf. $2015 ; 4670-4676$. 